\title{
Ground State Laser Cooling Using Electromagnetically Induced Transparency
}

\author{
Giovanna Morigi, ${ }^{1}$ Jürgen Eschner, ${ }^{2}$ and Christoph H. Keitel ${ }^{3}$ \\ ${ }^{1}$ Max-Planck Institut für Quantenoptik, D-85748 Garching, Germany \\ ${ }^{2}$ Institut für Experimentalphysik, University of Innsbruck, A-6020 Innsbruck, Austria \\ ${ }^{3}$ Theoretische Quantendynamik, Fakultät für Physik, University of Freiburg, D-79104 Freiburg, Germany
}

(Received 5 May 2000)

\begin{abstract}
A laser cooling method for trapped atoms is described which achieves ground state cooling by exploiting quantum interference in a driven $\Lambda$-shaped arrangement of atomic levels. The scheme is technically simpler than existing methods of sideband cooling, yet it can be significantly more efficient, in particular when several motional modes are involved, and it does not impose restrictions on the transition linewidth. We study the full quantum mechanical model of the cooling process for one motional degree of freedom and show that a rate equation provides a good approximation.
\end{abstract}

PACS numbers: 32.80.Pj, 03.67.Lx, 42.50.Gy, 42.50.Vk

Laser cooling has played a central role in the preparation of fundamental quantum mechanical atomic systems [1], for example, in experiments which study the quantum statistical properties of atoms [2] or which use trapped ions for processing information at the quantum level $[3,4]$. In the latter context, laser cooling of trapped ions to the ground state of the confining potential is a fundamental step in the preparation of the ion trap quantum computer [5]. Furthermore, the same techniques that allow ground state cooling are at the basis of coherent manipulation, i.e., gate operations, in quantum computation schemes with trapped ions. For both purposes, cooling and gate operations, the speed of the manipulation has become an important issue [6], because higher speed means that competing heating or decoherence due to coupling to the environment has less opportunity to perturb the desired processes.

Efficient ground state laser cooling of single trapped ions has been achieved using two-level sideband cooling [7] and Raman sideband cooling [8], and recently these methods have been transferred to two ions [9] and to atomic gases [10]. These techniques involve laser excitation of an atom with two internal levels $|g\rangle$ and $|e\rangle$, which in the case of Raman sideband cooling is designed from a $\Lambda$-shaped three-level atom by Raman coupling [11,12]. Both techniques rely on several conditions [13]: (i) the motional spectrum of the system has equidistant levels $|n\rangle$, which is true when the particle (or particles) are trapped in a harmonic potential; (ii) the amplitude of the oscillations of the trapped particles is much smaller than the wavelength of the cooling laser ("Lamb-Dicke regime"); (iii) the linewidth $\gamma$ of the internal transition is much smaller than the distance between any pair of motional energy levels ("strong confinement"). For the case of a single particle confined in a harmonic oscillator potential with frequency $\nu$, the strong confinement condition is $\gamma \ll \nu$.

Under these conditions it is possible to selectively excite sidebands of the optical resonance, i.e., transitions corresponding to a fixed change of the vibrational quantum number $n$ to $n^{\prime}$, by tuning the laser into resonance with that transition, while all other transitions are well off resonance and thus only negligibly excited. Specifically, for sideband cooling transitions $|g, n\rangle \rightarrow|e, n-1\rangle$ are induced by tuning the laser to $\omega_{a}-\nu$, i.e., to the "red sideband" of the bare atomic resonance at frequency $\omega_{a}$. When a spontaneous decay from $|e\rangle$ to $|g\rangle$ takes place or, in the case of Raman sideband cooling, when the atom is optically pumped back from $|e\rangle$ to $|g\rangle$, this decay occurs with highest probability on the transition $|e, n-1\rangle \rightarrow|g, n-1\rangle$ due to the Lamb-Dicke condition. Thus in one fluorescence cycle the system is cooled, on average, by one vibrational quantum. The cooling limit is determined by the equilibrium between these cooling cycles and heating processes. Heating is induced by off-resonant excitation of the $|g, n\rangle \rightarrow|e, n\rangle$ "carrier" transition followed by a $|e, n\rangle$ to $|g, n+1\rangle$ spontaneous emission event, or by excitation of a $|g, n\rangle \rightarrow$ $|e, n+1\rangle$ "blue sideband" transition. Since the selective excitation of the $|g, n\rangle \rightarrow|e, n-1\rangle$ sideband is at the basis of this technique, this imposes a limitation on the intensity of the cooling laser and thus also on the cooling speed. In particular, high laser intensity leads to increased off-resonant excitation of carrier transitions which limits the final ground state occupation of the cooling process.

In this paper we describe a method for ground state cooling of atoms with a multilevel structure which eliminates the carrier excitation by electromagnetically induced transparency [14]. The technique is based on continuous laser excitation and has several advantages over both two-level and Raman sideband cooling. Unlike in two-level sideband cooling, no strong confinement is required; instead two dipole-allowed transitions are used, neither of which has to fulfill the relation $\gamma \ll \nu$. Unlike Raman sideband cooling which involves an additional repumping laser, only two lasers are needed in our method. Finally, as will be shown, by canceling the carrier transition our scheme provides more efficient ground state cooling than sideband cooling methods, in particular for simultaneous cooling of several modes of vibration. This work extends previous analyses of laser cooling in a three-level atomic system [11,12], which, however, focused on different cooling mechanisms, as will be discussed below. 
Electromagnetically induced transparency (EIT) arises in three-level (or multilevel) systems and consists of the cancellation of the absorption on one transition induced by simultaneous coherent driving of another transition. The phenomenon is also called "coherent population trapping" [15] or "dark resonance" and has been demonstrated in many systems [16] including single trapped ions [17]. It belongs to a large class of quantum interference effects in multilevel systems [18] and can be understood as a destructive interference of the two pathways to the excited level [19]. It is also at the basis of velocity selective coherent population trapping, a laser cooling method for free atoms which achieves subrecoil temperatures [20]. Here we use this situation to suppress absorption on the $|g, n\rangle \rightarrow|e, n\rangle$ transition, while enhancing the absorption on the $|g, n\rangle \rightarrow|e, n-1\rangle$ sideband transition, thus decreasing the heating and increasing the cooling rate.

Let us for the moment neglect the motional degrees of freedom and consider a three-level atom with ground state $|g\rangle$, stable or metastable state $|r\rangle$, and excited state $|e\rangle$ in $\Lambda$ configuration as shown in Fig. 1. State $|e\rangle$ has linewidth $\gamma$ and is coupled to both $|g\rangle$ and $|r\rangle$ by dipole transitions. The transition $|r\rangle \rightarrow|e\rangle$ is excited by an intense "coupling" laser field of frequency $\omega_{r}$, Rabi frequency $\Omega_{r}$, and detuning $\Delta_{r}=\omega_{r}-\omega_{r e}$, where $\omega_{r e}$ is the frequency of the bare atomic transition $|r\rangle \rightarrow|e\rangle$. The absorption spectrum observed by exciting the transition $|g\rangle \rightarrow|e\rangle$ with another "cooling" laser at frequency $\omega_{g e}+\Delta_{g}$ and Rabi frequency $\Omega_{g}$ is described by a Fano-like profile [19], whose zero corresponds to the case $\Delta_{g}=\Delta_{r}$ and which is asymmetric for $\Delta_{r} \neq 0$; see Fig. 1 . The same spectrum describes the rate at which photons are scattered from state $|e\rangle$, and one can infer from it the cooling effect of the laser excitation on the ion [21].

In the case $\Delta_{r}>0$, which is displayed in Fig. 1, the two components of the absorption spectrum, i.e., the broad resonance at $\Delta_{g} \simeq 0$ with linewidth $\gamma^{\prime \prime} \simeq \gamma$ and the nar-
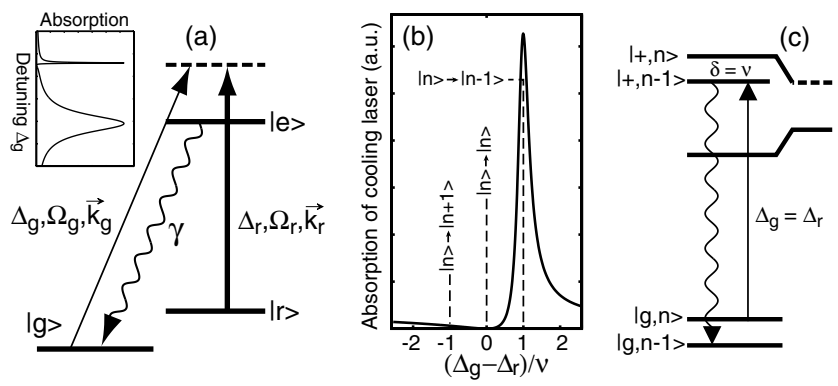

FIG. 1. (a) Levels and transitions of the cooling scheme (found in many species used for ion trapping). The inset shows schematically the absorption rate on $|g\rangle \rightarrow|e\rangle$ when the atom is strongly excited above resonance on $|r\rangle \rightarrow|e\rangle$. (b) Absorption of cooling laser around $\Delta_{g}=\Delta_{r}$ (solid line) and probabilities of carrier $(|n\rangle \rightarrow|n\rangle)$ and sideband $(|n\rangle \rightarrow|n \pm 1\rangle)$ transitions when $\Delta_{g}=\Delta_{r}$ (dashed lines). (c) The cooling laser excites resonantly transitions from $|g, n\rangle$ to the narrow dressed state [22] denoted by $|+, n-1\rangle$. row resonance at $\Delta_{g} \simeq \Delta_{r}$ with linewidth $\gamma^{\prime} \ll \gamma$, correspond to the dressed states of the system atom + coupling laser [22]; see Fig. 1c. These dressed states, and hence the maxima of the narrow and the broad curves, are shifted from $\Delta_{r}$ by $+\delta$ and $-\Delta_{r}-\delta$, respectively, with

$$
\delta=\left(\sqrt{\Delta_{r}^{2}+\Omega_{r}^{2}}-\left|\Delta_{r}\right|\right) / 2
$$

being the ac Stark shift induced by the coupling laser.

With the harmonic motion taken into account, the zero of the Fano-like profile at $\Delta_{g}=\Delta_{r}$ corresponds to the $|g, n\rangle \rightarrow|e, n\rangle$ transition which is therefore canceled. Then, by choosing $\Delta_{r}>0$ and a suitable Rabi frequency $\Omega_{r}$, the spectrum can be designed such that the $|g, n\rangle \rightarrow|e, n-1\rangle$ (red) sideband corresponds to the maximum of the narrow resonance, whereas the blue sideband falls into the region of the spectrum of small excitation probability, as shown in Fig. 1b. The condition on the laser parameters for enhancing the red-sideband absorption while eliminating the carrier is therefore

$$
\Delta_{g}=\Delta_{r} ; \quad \delta \simeq \nu .
$$

The laser parameters of Eq. (2) are easily achievable in single ion experiments [4,8] where typically $\gamma \sim 2 \pi \times$ $20 \mathrm{MHz}$ and $\nu \sim 2 \pi \times 1 \mathrm{MHz}$.

Note that the detunings are different from Raman sideband cooling (RSC) where $\Delta_{g}=\Delta_{r}-\nu$. Furthermore, here both lasers must be blue detuned from their respective atomic resonances, whereas in RSC they can be tuned either both below or both above resonance. Moreover, in RSC the bare states $|g\rangle,|r\rangle$ are coupled under saturation to $|e\rangle$ (this is the situation described in $[11,12]$ ), whereas in the new cooling scheme both multiple scattering on the transition $|r\rangle \rightarrow|e\rangle$ and the quantum interference at $\Delta_{g}=\Delta_{r}$ are crucial for the cooling process, which is therefore adequately described by transitions between state $|g\rangle$ and the two dressed states; see Fig. 1c.

The mechanism is theoretically modeled as follows. We start with the master equation for the full three-level system and one motional degree of freedom. In the Lamb-Dicke regime, the master equation can be reduced to a rate equation projected on the internal state $|g, n\rangle$ provided that $\Omega_{g} \ll \Omega_{r}$ and that the transition to the narrow dressed state of linewidth $\gamma^{\prime}$ is not saturated, i.e., $\Omega_{g} \ll \sqrt{\gamma \gamma^{\prime}}$. Then, in second order of the expansion in $\Omega_{g} / \sqrt{\gamma \gamma^{\prime}}$ the dynamics is described by an equation for the populations $P(n)$ of the vibrational number states $|n\rangle$ [23]:

$$
\begin{aligned}
\frac{d}{d t} P(n)=\eta^{2}\left\{A_{-}[(n+1) P(n+1)-n P(n)]\right. \\
\left.+A_{+}[n P(n-1)-(n+1) P(n)]\right\} .
\end{aligned}
$$

Here $\eta_{\vec{b}}$ is the Lamb-Dicke parameter, defined as $\eta=$ $\left|\vec{k}_{g}-\vec{k}_{r}\right| a_{0}$ with $a_{0}$ rms size of the ground state of the harmonic oscillator and $\vec{k}_{g}\left(\vec{k}_{r}\right)$ cooling (coupling) laser wave vector [24]. The coefficients $A_{ \pm}$have the form 


$$
A_{ \pm}=\frac{\Omega_{g}^{2}}{\gamma} \frac{\gamma^{2} \nu^{2}}{\gamma^{2} \nu^{2}+4\left[\Omega_{r}^{2} / 4-\nu(\nu \mp \Delta)\right]^{2}},
$$

where we have set $\Delta_{r}=\Delta_{g}=\Delta$. Note that while Eq. (3) has the same structure as the rate equation that describes cooling of a two-level atom in the Lamb-Dicke regime [13], the particular form of $A_{ \pm}$in Eq. (4) contains the full quantum interference around $\Delta_{g}=\Delta_{r}$. Solving Eq. (3) for the steady state value $\left\langle n_{S}\right\rangle$ of the mean vibrational quantum number $\langle n\rangle=\sum n P(n)$ we get

$$
\left\langle n_{S}\right\rangle=\frac{A_{+}}{A_{-}-A_{+}}=\frac{\gamma^{2} \nu^{2}+4\left[\Omega_{r}^{2} / 4-\nu(\nu+\Delta)\right]^{2}}{4 \Delta \nu\left(\Omega_{r}^{2}-4 \nu^{2}\right)} .
$$

Equation (5) has a pole $\left(A_{+}=A_{-}\right)$at $\Delta=0$, where the spectrum is symmetric, and at $\Omega_{r}=2 \nu$, where the value of the absorption spectrum at the two frequencies $\Delta_{g} \pm \nu$ is the same. For properly chosen parameters, however, a value of $\left\langle n_{S}\right\rangle$ close to zero can be reached, as shown in the example of Fig. 2. The optimum value $\left\langle n_{S}\right\rangle=(\gamma / 4 \Delta)^{2}$ is found when the second term in the numerator of Eq. (5) vanishes, which corresponds precisely to the condition $\delta=\nu$ in Eq. (2).

From Eq. (3) the time dependence of $\langle n\rangle$ follows:

$$
\langle\dot{n}\rangle=-\eta^{2}\left(A_{-}-A_{+}\right)\langle n\rangle+\eta^{2} A_{+},
$$

where $\eta^{2}\left(A_{-}-A_{+}\right)$is the cooling rate. This rate together with $\left\langle n_{S}\right\rangle$ determines the efficiency of the cooling technique which is compared to conventional sideband cooling below.

The dynamics of the full system for any set of parameters can be calculated with a quantum Monte Carlo simulation [25]. In Fig. 3 we plot the result of such a calculation and compare it to the rate equation solution of Eq. (6). We see that the rate equation provides a good description of the cooling. In this example, $99 \%$ occupation of the ground state is achieved.
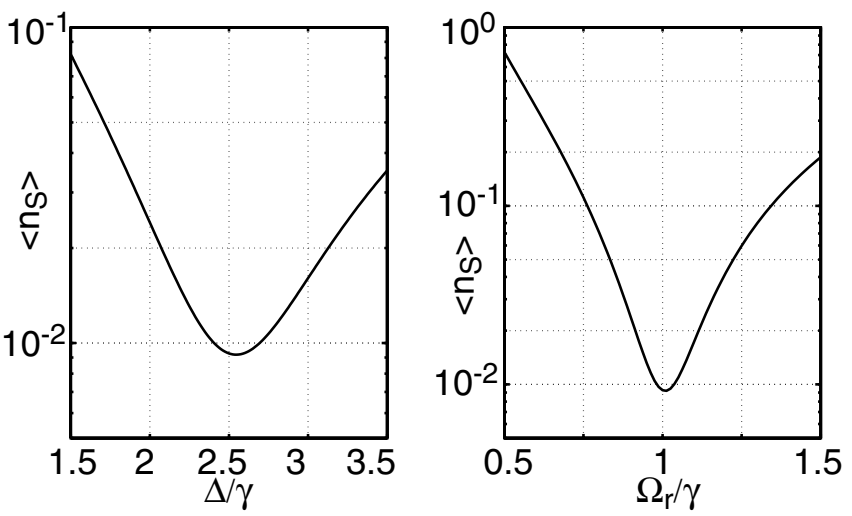

FIG. 2. Cooling limit $\left\langle n_{S}\right\rangle$ [Eq. (5)] as a function of detuning $\Delta / \gamma$ (left) and Rabi frequency of the coupling laser $\Omega_{r} / \gamma$ (right). The other parameters are $\nu=\gamma / 10, \Omega_{r}=\gamma$ (left), and $\Delta=2.5 \gamma$ (right).
In order to compare our EIT cooling scheme with conventional two-level sideband cooling, we use the results of the respective rate equations for both schemes and we allow for an angle $\phi$ between the motional axis and the direction of the laser beam (of $\vec{k}_{g}-\vec{k}_{r}$ in the case of EIT cooling). We denote by $A_{ \pm}^{\mathrm{SC}}$ the coefficients for sideband cooling corresponding to Eq. (4); cf. [13]. Assuming a linewidth $\gamma_{\mathrm{SC}}=\gamma^{\prime}$ for the sideband cooling transition, and the same degree of saturation in both schemes, i.e., $\left(\Omega_{\mathrm{SC}} / \gamma_{\mathrm{SC}}\right)^{2}=\Omega_{g}^{2} / \gamma \gamma^{\prime}$, the following relation holds:

$$
A_{ \pm}^{\mathrm{SC}}=A_{ \pm}+\frac{\Omega_{\mathrm{SC}}^{2}}{\gamma_{\mathrm{SC}}}\left(\frac{\alpha}{\cos ^{2} \phi}\right) \frac{\gamma_{\mathrm{SC}}^{2}}{\gamma_{\mathrm{SC}}^{2}+4 \nu^{2}} .
$$

Here we have used that $\gamma^{\prime} \approx \gamma \frac{\nu}{\Delta}$ for our conditions $\delta=$ $\nu \ll \Delta, \Omega_{r}$ [22], and $\alpha=\int_{-1}^{1} d u N(u) u^{2}$ with $N(\cos \vartheta)$ being the azimuthal dipole pattern of spontaneous emission on the sideband cooling transition. The additive term in Eq. (7) highlights that the difference between sideband cooling and EIT cooling lies in the heating that accompanies carrier absorption, which in EIT cooling is canceled. This leads to the remarkable result that in contrast to any other cooling scheme, in EIT cooling the theoretical limit $\left\langle n_{S}\right\rangle$ does not depend on the angle between the direction of the laser beams (of $\vec{k}_{g}-\vec{k}_{r}$ ) and the motional axis. For the typical condition of sideband cooling $\gamma_{\mathrm{SC}} \ll \nu$ we find, using Eq. (5),

$$
\left\langle n_{S}\right\rangle_{\mathrm{EIT}}=\frac{\left\langle n_{S}\right\rangle_{\mathrm{SC}}}{1+\frac{4 \alpha}{\cos ^{2} \phi}} .
$$

Thus, given the same cooling rate, $\left\langle n_{S}\right\rangle_{\mathrm{EIT}}<\left\langle n_{S}\right\rangle_{5}$ SC. The factor between the two steady state values is $\frac{5}{29}$ for all three motional degrees of freedom, assuming the ion is cooled in three dimensions by a single pair of laser beams, $\alpha=\frac{2}{5}$ [13], and $\vec{k}_{g}-\vec{k}_{r}$ has the same angle with all axes

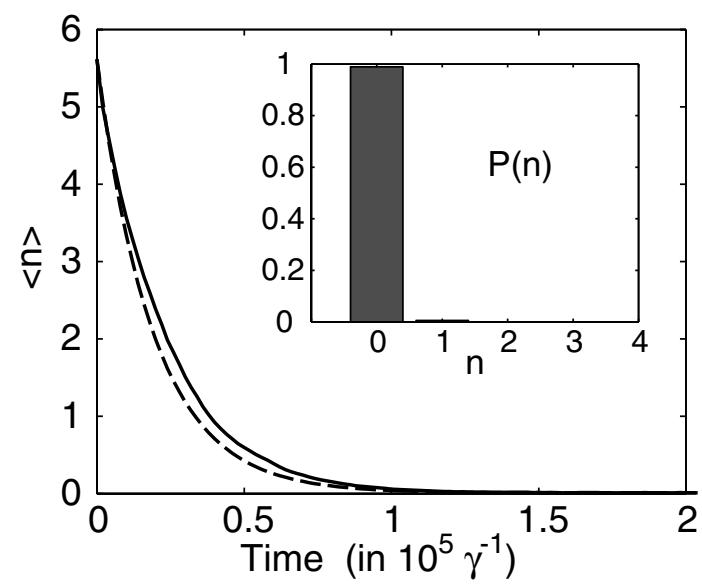

FIG. 3. $\langle n\rangle$ as a function of time calculated with full Monte Carlo simulation (solid line) and rate equation (dashed line). Parameters are $\Omega_{r}=\gamma, \Omega_{g}=\gamma / 20, \nu=\gamma / 10, \eta=0.145$, $\Delta_{g}=\Delta_{r}=2.5 \gamma$. The atomic parameters are for the $\left(S_{1 / 2}, P_{1 / 2}\right.$, $\left.D_{3 / 2}\right)-\Lambda$-system of a $\mathrm{Ca}^{+}$ion [4] where $\gamma=2 \pi \times 20 \mathrm{MHz}$. Inset: Steady state distribution $P(n)$. 
of the trap. It becomes even smaller when $\vec{k}_{g}-\vec{k}_{r}$ is at a large angle with the motional axis. This makes EIT cooling a significantly more efficient technique in many typical experimental situations [26]. Moreover, numerical studies show that the efficiency can be increased further if the cooling laser is tuned not exactly to the dark resonance but slightly above such that the combined heating of carrier and blue sideband transitions is minimized.

In conclusion, we have presented a laser cooling technique for trapped particles which exploits quantum interference, or electromagnetically induced transparency, in a three-level atom. By appropriately designing the absorption profile with a strong coupling laser, the cooling transitions induced by a cooling laser are enhanced while heating by resonant absorption is suppressed. The method is not based on the strong confinement condition and requires only two continuous lasers. We have derived a simple model for describing the cooling process and shown that it is in good agreement with a full quantum Monte Carlo treatment. With the same cooling rate as in conventional sideband cooling, much higher ground state occupation is achieved by our method, in particular, if three-dimensional cooling is considered. The technical requirements, two lasers with a well-controlled frequency difference, are met by most existing single ion experiments, and they are less stringent than for both Raman sideband cooling and ordinary two-level sideband cooling. Furthermore, the method is insensitive to laser frequency fluctuations as long as the laser linewidth is small compared to the trap frequency.

Simultaneous cooling in three dimensions can be achieved with this method if the trap frequencies along the axes are similar, so that the red sideband for each oscillator falls into the neighborhood of the maximum of the narrow resonance. Similarly, the method can be applied to simultaneously cool several axial modes of an $\mathrm{N}$-ion crystal in a linear trap, or atoms in anharmonic traps, where the energies of the motional states are not equidistant. An extension would be to use an atom with more than three levels, where multiple dark resonances occur (see, e.g., [17]) and to design the absorption spectrum such that both the carrier and the blue sideband transition vanish.

Following the ideas of this proposal, the method has meanwhile been experimentally demonstrated [27].

We thank J. I. Cirac, P. Lambropoulos, D. Leibfried, C. Roos, F. Schmidt-Kaler, H. Walther, and P. Zoller for many stimulating discussions, and acknowledge support by the European Commission (TMR networks ERB-FMRXCT96-0077 and ERB-FMRX-CT96-0087; Marie Curie Program) and the German Science Foundation (SFB 276). C.H.K. is thankful for the hospitality at Innsbruck University.
[1] S. Chu, Rev. Mod. Phys. 70, 685 (1998); C. CohenTannoudji, ibid. 70, 707 (1998); W. D. Phillips, ibid. 70, 721 (1998).

[2] A. J. Kerman et al., Phys. Rev. Lett. 84, 439 (2000); M. Morinaga et al., Phys. Rev. Lett. 83, 4037 (1999).

[3] D. J. Wineland et al., J. Res. Natl. Inst. Stand. Technol. 103, 259 (1998); R. J. Hughes et al., Fortschr. Phys. 46, 329 (1998).

[4] H. C. Nägerl et al., Appl. Phys. B 66, 603 (1998).

[5] J. I. Cirac and P. Zoller, Phys. Rev. Lett. 74, 4091 (1995).

[6] A. Steane et al., Phys. Rev. A 62, 042305 (2000).

[7] F. Diedrich et al., Phys. Rev. Lett. 62, 403 (1989); Ch. Roos et al., Phys. Rev. Lett. 83, 4713 (1999).

[8] C. Monroe et al., Phys. Rev. Lett. 75, 4011 (1995).

[9] B.E. King et al., Phys. Rev. Lett. 81, 1525 (1998); F. Schmidt-Kaler et al., quant-ph/0003096.

[10] H. Perrin et al., Europhys. Lett. 42, 395 (1998); S.E. Hamann et al., Phys. Rev. Lett. 80, 4149 (1998); V. Vuletić et al., Phys. Rev. Lett. 81, 5768 (1998).

[11] M. Lindberg and J. Javanainen, J. Opt. Soc. Am. B 3, 1008 (1986).

[12] I. Marzoli et al., Phys. Rev. A 49, 2771 (1994).

[13] S. Stenholm, Rev. Mod. Phys. 58, 699 (1986).

[14] E. Arimondo and G. Orriols, Lett. Nuovo Cimento 17, 333 (1976); S. E. Harris, Phys. Today 50, No. 7, 36 (1997), and references therein.

[15] H. R. Gray et al., Opt. Lett. 3, 218 (1978); P. M. Radmore and P. L. Knight, J. Phys. B 15, 561 (1982).

[16] G. Alzetta et al., Nuovo Cimento Soc. Ital. Fis. 36B, 5 (1976); L. V. Hau et al., Nature (London) 397, 594 (1999); for a review see J.P. Marangos, J. Mod. Opt. 45, 471 (1998).

[17] G. Janik et al., J. Opt. Soc. Am. B 2, 1251 (1985); I. Siemers et al., Europhys. Lett. 18, 139 (1992).

[18] O. Kocharovskaya and Y. I. Khanin, JETP Lett. 48, 630 (1988); M. O. Scully and S. Y. Zhu, Science 281, 1973 (1998), and references therein.

[19] B. Lounis and C. Cohen-Tannoudji, J. Phys. II (France) 2, 579 (1992), and references therein.

[20] A. Aspect et al., Phys. Rev. Lett. 61, 826 (1988).

[21] W. Neuhauser et al., Phys. Rev. Lett. 41, 233 (1978).

[22] C. Cohen-Tannoudji et al., Atom-Photon Interactions (Wiley, New York, 1992).

[23] G. Morigi et al. (unpublished).

[24] Note that for parallel wave vectors of equal magnitude all transitions to $|e\rangle$, including the sideband transitions, are suppressed, whereas for antiparallel wave vectors the carrier excitation vanishes as desired and the two wave vectors add up on the sidebands. This effect is known from Raman sideband cooling [8,11].

[25] J. Dalibard et al., Phys. Rev. Lett. 68, 580 (1992).

[26] F. Schmidt-Kaler (private communication).

[27] C. F. Roos, D. Leibfried, A. Mundt, F. Schmidt-Kaler, J. Eschner, and R. Blatt, quant-ph/0009034. 